\section{Morbus Behçet: Welche pathogenetische Rolle spielen neutrophile Netzwerke?}

Le Joncour $A$ et al. Critical role of neutrophil extracellular traps (NETs) in patients with Behcet's disease. Ann Rheum Dis 2019; 78 (9): 1274-1282. doi: 10.1136/annrheumdis-2018-214335

Thrombosen stellen eine - nicht selten lebensbedrohliche - Komplikation des Morbus Behçet dar. Ähnlich wie die Pathophysiologie dieser immunologisch-inflammatorischen, chronischen systemischen Vaskulitis sind auch die exakten Pathomechanismen der Thromboseneigung bislang nur unvollständig verstanden. Möglicherweise spielen sogenannte Neutrophil Extrazellular Traps (NETs) hierbei eine wichtige Rolle, berichten nun französische Forscher.

Die Neutrophilen von Patienten mit einem Morbus Behçet sind hyperaktiviert und zei- gen eine verstärkte Phagozytoseaktivität und Superoxidproduktion, erläutern die Wissenschaftler. Unter inflammatorischen oder infektiösen Bedingungen sind Neutrophile in der Lage, über einen als NETose bezeichneten Zelltod-Mechanismus NETs zu bilden. Diese thrombogenen Netzwerke bestehen aus ausgestoßener zellfreier DNA, Histonen sowie granulären Komponenten inklusive antimikrobiell wirksamer Peptide und Proteasen. Im Rahmen der NETose kommt es zu einer Kalziummobilisation, zur Bildung reaktiver Sauerstoffspezies, zur enzymatischen nukleären Delobulierung mittels Myeloperoxidase sowie zur Modifikation der neutrophilen Elastase bzw. des Chromatins durch Histon-Zitrullinierung mittels Peptidyl-Arginin-Deiminase (PAD4). NETs scheinen eine wichtige pathogenetische Rolle bei anderen autoinflammatorischen und autoimmunen Erkrankungen, bei der Thrombusbildung und -progression bei der tiefen Venenthrombose sowie bei arteriellen Prozessen (Stroke, Myokardinfarkt) zu spielen. Welche Bedeutung sie beim Morbus Behçet haben, untersuchten die Forscher nun anhand von Serumproben von 73 Betroffenen mit einem klinisch/serologisch aktiven ( $n=28)$ bzw. inaktiven $(n=45)$ Morbus Behçet. Das Vergleichskollektiv bildeten 15 gesunde Personen. Die Laboranalysen umfassten die Objektivierung verschiedener NET-Komponenten, bspw. der zellfreien DNA sowie der Myeloperoxidase.

\section{Ergebnisse}

Patienten mit einem aktiven Morbus Behçet wiesen sowohl im Vergleich zu Patienten mit einer inaktiven Erkrankung als auch im Vergleich zu Gesunden signifikant höhere Serumspiegel zellfreier DNA sowie von Myeloperoxidase-DNA-Komplexen auf. Patienten mit vaskulärer Beteiligung zeigten im Vergleich zu Patienten ohne Gefäßsymptome ebenfalls höhere Spiegel beider Parameter. Die von Behçet-Patienten isolierten Neutrophilen produzierten im Vergleich zu den Neutrophilen von Gesunden verstärkt reaktive Sauerstoffspezies und befanden sich in einem präaktivierten Zustand: Sie initiierten deutlich häufiger eine spontane NETose. Ferner wiesen die Forscher bei den Behçet-Patienten eine Hyperkoagulabilität nach: Ihr Plasma zeigte eine verstärkte Thrombinbildung, die positiv mit dem Spiegel zellfreier DNA sowie von
Myeloperoxidase-DNA-Komplexen korrelierte. Behandelten die Forscher das Plasma mit DNAse, verringerte sich bei den BehçetPatienten - nicht jedoch bei den Gesunden - die Thrombinbildung. Diese Beobachtung spricht nach Ansicht der Forscher dafür, dass NET-Komponenten beim Morbus Behçet eine prokoagulatorische Wirkung entfalten. Abschließend untersuchten die Wissenschaftler Aortenbiopsien von 3 symptomatischen Behçet-Patienten immunhistologisch auf NETs. Das Ergebnis: In befallenen Gefäßarealen mit vaskulitischen bzw. mikrothrombotischen Läsionen sowie in Arealen mit Neutrophileninfiltration stellten sich gehäuft NET-bildende Neutrophile dar.

FAZIT

NETs und ihre Komponenten sind bei Patienten mit einem Morbus Behçet verstärkt nachweisbar, schlussfolgern die Autoren. Sie vermuten, dass diese neutrophilen Netzwerke eine wesentliche Rolle bei der Pathogenese der Gefäßmanifestationen sowie der Hyperkoagulabilität spielen. NETs stellen ihrer Einschätzung zu Folge wahrscheinlich nicht nur einen Biomarker für den Erkrankungsschweregrad dar, sondern - so ihre Hoffnung - der NETose-Prozess eignet sich möglicherweise auch als potenzielles Therapieziel.

Dr. med. Judith Lorenz, Künzell 